UCSBTH-92-11

hepth@xxx/9203083

March, 1992

\title{
Rotating Dilaton Black Holes
}

\author{
James H. Horne and Gary T. Horowitz \\ Department of Physics \\ University of California \\ Santa Barbara CA 93106-9530 \\ jhh@cosmic.physics.ucsb.edu \\ gary@cosmic.physics.ucsb.edu
}

\begin{abstract}
It is shown that an arbitrarily small amount of angular momentum can qualitatively change the properties of extremal charged black holes coupled to a dilaton. In addition, the gyromagnetic ratio of these black holes is computed and an exact rotating black string solution is presented.
\end{abstract}




\section{Introduction}

All stationary black holes in general relativity coupled to electromagnetism are described by the Kerr-Newman solutions. These solutions contain three parameters, the mass $M$, charge $Q$, and angular momentum $J$, which satisfy the inequality $M^{2} \geq Q^{2}+J^{2} / M^{2}$. (When this inequality is violated, the event horizon disappears and the spacetime describes a naked singularity.) It has been known for a long time that if one minimally couples certain additional matter fields such as scalars, neutrinos or charged fermions, then the black hole solutions do not change. It has recently become clear that if one couples gravity to more complicated forms of matter, then the black hole solutions do change. In particular, if one couples to an SU(2) Yang-Mills theory, new black hole solutions exist [1] (although they have been shown to be unstable [2]). If one includes a Higgs field as well as the gauge field, there are additional solutions which can be interpreted as black holes inside nonabelian monopoles [3].

In this paper we will focus on perhaps the simplest extension of the standard matter - gravity coupled to a Maxwell field and a dilaton. The action is

$$
S=\int d^{4} x \sqrt{-g}\left(-R+2(\nabla \Phi)^{2}+e^{-2 \alpha \Phi} F^{2}\right)
$$

where $\alpha$ is a free parameter which governs the strength of the coupling of the dilaton to the Maxwell field*. When $\alpha=0$, the action reduces to the usual Einstein-Maxwell-scalar theory. When $\alpha=1$, the action (1) is part of the low energy action of string theory. However, we will consider this theory for all values of $\alpha$. Since changing the sign of $\alpha$ is equivalent to changing the sign of $\Phi$, it is sufficient to consider only $\alpha \geq 0$.

Nonrotating charged black hole solutions of (1) have been found [4,5] and are reviewed below. Certain qualitative features of the solutions turn out to be independent of $\alpha$. For example, for a given mass, there is always a maximum charge that can be carried by the black hole. If the charge is less than this extremal value, there is a regular event horizon. However, other qualitative features depend crucially on $\alpha$. For example, for $\alpha \neq 0$ there is no inner horizon. More importantly, in the extremal limit, the area $A$ of the event horizon and the surface gravity $\kappa$ are discontinuous functions of $\alpha .^{\dagger}$ This has led to the view [7] that properties of black holes are not universal, but depend on the details of the matter lagrangian. In [7], it was shown that for $\alpha>1$, infinite potential barriers form around

* In recent papers, this parameter has been called $a$. We adopt a different notation here to avoid confusion with the standard angular momentum parameter for rotating black holes.

$\dagger$ In the thermodynamic description, the entropy is simply $S=\frac{1}{4} A$ and the temperature is $T=\frac{\kappa}{2 \pi}$. Since it has been argued that the thermodynamic interpretation breaks down near the extremal limit [6], we will focus on the geometrically well-defined quantities $A$ and $\kappa$. 
extremal black holes. This led to the interpretation that the extremal black holes behave like elementary particles.

However, the black hole solutions which have been studied so far represent only a special case of the general black hole solution since rotation has been ignored. To see whether qualitative properties of generic black holes depend on the matter content, one must consider rotating charged black holes. This is the subject of the present investigation. We have not yet found explicit solutions describing rotating charged black holes for arbitrary $\alpha$. However for $\alpha=\sqrt{3}$, the solutions are known [8]. By considering this one exact solution and some general arguments, we are led to the conclusion that at least in terms of properties such as the area of the event horizon and the surface gravity, rotating black holes do not depend qualitatively on $\alpha$. For example, for $\alpha=\sqrt{3}$, the area of the event horizon of an extremal black hole is simply proportional to the angular momentum,

$$
A=8 \pi|J| \text {. }
$$

Thus the area is nonzero in general, and vanishes only in the limit of no rotation. In addition, the surface gravity vanishes in the extremal limit whenever the angular momentum is nonzero. These properties are more analogous to the usual Kerr-Newman solution $(\alpha=0)$ than the nonrotating $\alpha=\sqrt{3}$ solution. Nevertheless, we will argue that when the quantum effects of particle creation are taken into account, the late time behavior of rotating dilaton black holes still resembles that of elementary particles rather than traditional black holes.

Although we do not yet have explicit rotating black hole solutions for all values of $\alpha$, we will present the solutions in the limit of slow rotation. This is sufficient to compute the gyromagnetic ratio of the black hole i.e., the ratio of the magnetic dipole moment to the angular momentum. This is of interest since in the Einstein-Maxwell theory, black holes have a gyromagnetic ratio corresponding to $g=2$ (the value for electrons) rather than $g=1$ (the value for classical matter). We will see that this is modified when $\alpha \neq 0$. Additionally, extended black hole or black string solutions to low energy string theory have recently been found in various dimensions $[9,10,11]$. We will present an exact rotating charged black string solution in five dimensions which is closely related to the rotating dilaton black hole with $\alpha=\sqrt{3}$.

We begin by reviewing the unrotating charged black hole solutions to (1). The field equations are

$$
\begin{gathered}
\nabla_{\mu}\left(e^{-2 \alpha \Phi} F^{\mu \nu}\right)=0 \\
\nabla^{2} \Phi+\frac{\alpha}{2} e^{-2 \alpha \Phi} F^{2}=0 \\
R_{\mu \nu}=2 \nabla_{\mu} \Phi \nabla_{\nu} \Phi+2 e^{-2 \alpha \Phi} F_{\mu \rho} F_{\nu} \rho-\frac{1}{2} g_{\mu \nu} e^{-2 \alpha \Phi} F^{2} .
\end{gathered}
$$

The spherically symmetric solutions take the form $[4,5]$

$$
d s^{2}=-\lambda^{2} d t^{2}+\frac{d r^{2}}{\lambda^{2}}+R^{2} d \Omega
$$




$$
\begin{gathered}
e^{2 \Phi}=\left(1-\frac{r_{-}}{r}\right)^{\frac{2 \alpha}{1+\alpha^{2}}} \\
F_{t r}=\frac{Q}{r^{2}} .
\end{gathered}
$$

where

$$
\lambda^{2}=\left(1-\frac{r_{+}}{r}\right)\left(1-\frac{r_{-}}{r}\right)^{\frac{1-\alpha^{2}}{1+\alpha^{2}}}
$$

and

$$
R=r\left(1-\frac{r_{-}}{r}\right)^{\frac{\alpha^{2}}{1+\alpha^{2}}} .
$$

The two free parameters $r_{+}, r_{-}$are related to the physical mass and charge by

$$
\begin{gathered}
M=\frac{r_{+}}{2}+\left(\frac{1-\alpha^{2}}{1+\alpha^{2}}\right) \frac{r_{-}}{2} \\
Q=\left(\frac{r_{+} r_{-}}{1+\alpha^{2}}\right)^{\frac{1}{2}} .
\end{gathered}
$$

When $\alpha=0$ this solution reduces to the standard Reissner-Nordström solution of EinsteinMaxwell theory. However, for $\alpha \neq 0$ the solution is qualitatively different. For all $\alpha$, the surface $r=r_{+}$is an event horizon. The surface $r=r_{-}$is a curvature singularity except for the case $\alpha=0$ when it is a nonsingular inner horizon*. Thus they describe black holes only when $r_{-}<r_{+}$. In the extremal limit, $r_{-}=r_{+}$, it is clear from (6) and (10) that for $\alpha \neq 0$ the area of the event horizon goes to zero. The surface gravity $\kappa$ is

$$
\kappa=\frac{1}{2 r_{+}}\left(1-\frac{r_{-}}{r_{+}}\right)^{\frac{1-\alpha^{2}}{1+\alpha^{2}}} .
$$

Thus, for $\alpha<1$ the surface gravity goes to zero in the extremal limit, for $\alpha=1$ it approaches a constant, and for $\alpha>1$ it diverges. Holzhey and Wilczek [7] have shown that for $\alpha>1$ as the black hole approaches its extremal limit, there are potential barriers outside the horizon which increase without bound. Thus nearly extremal black holes do not absorb low energy incoming radiation. In addition, any radiation which is absorbed by the black hole is likely to be radiated very quickly since the temperature is proportional to $\kappa$. The nearly extremal black holes thus act more like elementary particles than traditional black holes.

\section{Rotating Black Holes}

Let us now consider rotating black holes. When $\alpha=0$, the rotating charged solution

* This is consistent with the idea that the inner horizon is unstable in the Einstein-Maxwell theory. 
is the familiar Kerr-Newman solution

$$
\begin{aligned}
d s^{2}= & -\left(\frac{\Delta-a^{2} \sin ^{2} \theta}{\Sigma}\right) d t^{2}-\frac{2 a \sin ^{2} \theta\left(r^{2}+a^{2}-\Delta\right)}{\Sigma} d t d \phi \\
& +\left[\frac{\left(r^{2}+a^{2}\right)^{2}-\Delta a^{2} \sin ^{2} \theta}{\Sigma}\right] \sin ^{2} \theta d \phi^{2}+\frac{\Sigma}{\Delta} d r^{2}+\Sigma d \theta^{2}, \\
A_{t}= & \frac{Q r}{\Sigma}, \quad A_{\phi}=-\frac{a Q r \sin ^{2} \theta}{\Sigma}, \quad \Phi=0,
\end{aligned}
$$

where

$$
\begin{gathered}
\Sigma=r^{2}+a^{2} \cos ^{2} \theta, \\
\Delta=r^{2}+a^{2}+Q^{2}-2 M r,
\end{gathered}
$$

and $a$ is the angular momentum $J$ divided by the mass. This solution has both an event horizon and an inner horizon at the zeros of $\Delta$. Perhaps the key difference between a rotating and nonrotating black hole is the existence of an ergosphere. (For a general discussion, see [12]). In the Kerr-Newman solution, $g_{t t}>0$ in a region outside the event horizon. This allows energy to be extracted classically either by the Penrose process for point particles or by superradiant scattering for fields. However, the area of the event horizon cannot decrease in these classical processes. The area can be expressed as

$$
A=8 \pi\left[\sqrt{D^{2}+Q^{4} / 4}+\sqrt{D^{2}-J^{2}}\right]
$$

where $D^{2} \equiv M^{2}\left(M^{2}-Q^{2}\right)$. The extraction of energy also reduces the angular momentum in such a way that $A$ increases.

For any $\alpha$, the rotating uncharged black hole is simply the $Q=0$ limit of (14) and is called the Kerr solution. The Kerr metric has an event horizon at $r=M+\sqrt{M^{2}-a^{2}}$ and an inner horizon at $r=M-\sqrt{M^{2}-a^{2}}$. The extremal limit has finite area and zero surface gravity. We have seen that for $\alpha>1$ the extremal limit of the unrotating charged black hole has zero area and infinite surface gravity. Which of these two situations, zero rotation or zero charge, is likely to have qualitative properties similar to the general rotating charged black hole? First, consider a large uncharged black hole which is rotating near its extremal rate. Suppose we add a small amount of charge. Since the event horizon is large and stable, we do not expect the charge to drastically change the geometry in its vicinity. This suggests that extremal rotating black holes with small charge will resemble the uncharged black holes. In particular, the area of the event horizon should be large and the surface gravity should be small. Furthermore, the potential barriers outside the horizon should remain modest. Now, suppose we start with a nearly extremal unrotating black hole and try to add a small amount of angular momentum. In this case, the event horizon is very small and the potential barriers are very large. Thus one cannot add angular momentum without sending it in with a large amount of energy which can significantly 
alter the properties of the solution. Thus there is little reason to expect that black holes with small angular momentum will resemble the zero angular momentum limit.

\section{The Kaluza-Klein Solution}

To determine the behavior of extremal black holes with small angular momentum, as well as to verify the above arguments for large angular momentum, one needs to examine exact solutions. There is one value of $\alpha$ for which an exact solution is known: $\alpha=\sqrt{3}$ [8]. This is well within the $\alpha>1$ regime of [7] where the extremal black holes are supposed to behave like elementary particles. For this value of $\alpha$, the action (1) is simply the KaluzaKlein action which is obtained by dimensionally reducing the five dimensional vacuum Einstein action. In other words, given a five dimensional spacetime with a translational symmetry in a spacelike direction, define a four dimensional metric $g_{\mu \nu}$, vector potential $A_{\mu}$ and dilaton $\Phi$ by

$$
d s^{2}=e^{4 \Phi / \sqrt{3}}\left(d x^{5}+2 A_{\mu} d x^{\mu}\right)^{2}+e^{-2 \Phi / \sqrt{3}} g_{\mu \nu} d x^{\mu} d x^{\nu}
$$

Then the field equations (3), (4), and (5) are equivalent to the five dimensional vacuum Einstein equation.

As a result, it is straightforward to find exact solutions for $\alpha=\sqrt{3}$. One starts with a four dimensional vacuum solution of Einstein's equation, takes the product with $\mathbf{R}$ to obtain a five dimensional translationally invariant solution, and finally boosts the solution in the extra direction. When reinterpreted in four dimensions, the solution has nonzero charge and a nontrivial dilaton field [13]. Starting with the Schwarzschild solution, this procedure yields the charged black hole (6) for $\alpha=\sqrt{3}$. To obtain rotating black holes, one simply starts with the Kerr solution and repeats the same procedure*. The resulting metric is [8]

$$
\begin{aligned}
d s^{2}= & -\frac{1-Z}{B} d t^{2}-\frac{2 a Z \sin ^{2} \theta}{B \sqrt{1-v^{2}}} d t d \phi \\
& +\left[B\left(r^{2}+a^{2}\right)+a^{2} \sin ^{2} \theta \frac{Z}{B}\right] \sin ^{2} \theta d \phi^{2}+B \frac{\Sigma}{\Delta_{0}} d r^{2}+B \Sigma d \theta^{2},
\end{aligned}
$$

where

$$
B=\sqrt{1+\frac{v^{2} Z}{1-v^{2}}}, \quad Z=\frac{2 m r}{\Sigma}, \quad \Delta_{0}=r^{2}+a^{2}-2 m r
$$

and $m, a$ are the mass and rotation parameters of the original Kerr solution and $v$ is the velocity of the boost. $\Sigma$ is defined in (15). The Kaluza-Klein solution has a vector potential

$$
A_{t}=\frac{v}{2\left(1-v^{2}\right)} \frac{Z}{B^{2}}, \quad A_{\phi}=-a \sin ^{2} \theta \frac{v}{2 \sqrt{1-v^{2}}} \frac{Z}{B^{2}},
$$

* Starting instead with the charged but massless Kerr-Newman solution leads to a massive Kaluza-Klein solution with a naked singularity [14]. 
and a dilaton field

$$
\Phi=-\frac{\sqrt{3}}{2} \log B
$$

The physical mass $M$, charge $Q$, and angular momentum $J$ are given by

$$
\begin{gathered}
M=m\left(1+\frac{v^{2}}{2\left(1-v^{2}\right)}\right) \\
Q=\frac{m v}{1-v^{2}} \\
J=\frac{m a}{\sqrt{1-v^{2}}} .
\end{gathered}
$$

It is easy to see that if $v=0$, the solution reduces to the original Kerr solution. Furthermore, if $a=0$, the solution agrees with (6)-(10) if one sets $\alpha=\sqrt{3}$, identifies

$$
\begin{aligned}
& r_{+}=\frac{2 m}{1-v^{2}} \\
& r_{-}=\frac{2 m v^{2}}{1-v^{2}}
\end{aligned}
$$

and does a simple coordinate transformation $r \rightarrow r+r_{-}$in (6)-(10). Notice that the extremal limit $r_{-} \rightarrow r_{+}$of the unrotating black hole corresponds, from the five dimensional viewpoint, to boosting Schwarzschild cross $\mathbf{R}$ to the speed of light $v=1$ while taking the unboosted mass $m$ to zero so that the limit is well defined. In particular, the infinite potential barriers that arise outside the horizon can be viewed as a result of boosting the finite barriers outside Schwarzschild.

The first thing to note about the rotating dilaton black hole (19) is that the causal structure is very similar to the Kerr solution. The metric appears singular at the zeros of $\Sigma$ and $\Delta_{0}$. The first turns out to be a true curvature singularity at $r=0, \theta=\pi / 2$ (the ring singularity) and the second is a coordinate effect associated with regular horizons. There is an event horizon at $r=m+\sqrt{m^{2}-a^{2}}$ and a nonsingular inner horizon at $r=m-\sqrt{m^{2}-a^{2}}$. Thus in the presense of rotation, the dilaton does not destroy the inner horizon. The extremal limit corresponds to $m^{2}=a^{2}$. (Notice that this condition is independent of $v$ - an extremal black hole remains extremal under the boost.) The extremal limit can be reexpressed as $J^{2}=C^{2}$ where $C \equiv m^{2} / \sqrt{1-v^{2}}$ depends on $M$ and $Q$, but is independent of the angular momentum $J$. It is easy to show that for a general black hole, the area of the event horizon is

$$
A=8 \pi\left[C+\sqrt{C^{2}-J^{2}}\right] .
$$

This shows that in the extremal limit, the area is simply proportional to the angular momentum: $A=8 \pi|J|$. This clearly demonstrates how the earlier result that the area vanishes for nonrotating black holes is modified by rotation. Although the area is nonzero 
in general, it clearly has a different dependence on the physical parameters $M, Q, J$ than the Kerr-Newman solution (17).

Next we consider the surface gravity of the black hole. This is [8]

$$
\kappa=\frac{\sqrt{\left(1-v^{2}\right)\left(m^{2}-a^{2}\right)}}{2 m\left[m+\sqrt{m^{2}-a^{2}}\right]} .
$$

If the angular momentum is nonzero, it follows immediately from (28) that in the extremal limit the surface gravity goes to zero. For the nonrotating black hole, the surface gravity reduces to $\kappa=\sqrt{1-v^{2}} / 4 m$ which agrees with (13) after using eq. (26) and setting $\alpha=\sqrt{3}$. In this case the surface gravity clearly diverges in the extremal limit. Thus one can view the surface gravity of the extremal black hole (somewhat heuristically) as being given by $\kappa=\delta(J)$.

Finally we consider the angular velocity of the horizon $\Omega$. This is defined by the condition that the Killing vector $(\partial / \partial t)+\Omega(\partial / \partial \phi)$ is null at the event horizon. For the Kaluza-Klein black hole (19), the angular velocity is [8]

$$
\Omega=\frac{a \sqrt{\left(1-v^{2}\right)}}{2 m\left[m+\sqrt{m^{2}-a^{2}}\right]} .
$$

This also exhibits an interesting discontinuity. If one considers nonextremal black holes and takes the limit as the angular momentum goes to zero, then $\Omega$ goes to zero as expected. However, if one considers extremal black holes and takes the limit as a goes to zero (keeping the physical mass $M$ fixed) one finds that $\Omega$ diverges. This is because the horizon is shrinking to zero size as the angular momentum decreases.

We now briefly consider the question of what is the endpoint of Hawking evaporation if one starts with a nonextremal black hole in this theory. Let us first consider a nonrotating black hole with initial mass $M$ and charge $Q$. Since the lagrangian (1) does not include any charged particles, it is clear that the charge cannot be radiated away. The mass will decrease toward the extremal value and the temperature will increase. However the potential barriers outside the black hole will also increase. It seems likely that the black hole only asymptotically approaches its extremal limit. It is amusing to consider this from the standpoint of the five dimensional theory. The five dimensional theory is more general than (1) because modes depending on the extra dimension correspond to massive charged fields in the four dimensional theory. As we have seen, a charged black hole is simply a boosted form of Schwarzschild cross $\mathbf{R}$. If the radius of the compact direction is much smaller than the wavelength of the Hawking radiation, then the momentum in this direction cannot change. (This is the five dimensional analog of the fact that the charge is constant.) However the mass will decrease. In order to conserve momentum it must speed up. The temperature increases and hence the wavelength of the emitted radiation decreases. This process continues until the wavelength is of order the size of the compact 
direction at which point the momentum can change. However, at this point the potential barriers are so large that it is unlikely that the black hole will be able to completely radiate away its momentum.

Now we include angular momentum. There are two basic facts about the quantum mechanics of rotating black holes. The first is that Hawking radiation carries away angular momentum. The ratio of angular momentum to energy carried away depends on the spin of the matter field and increases with increasing spin [15]. The second fact is that rotating black holes are never quantum mechanically stable. Even at zero temperature there is radiation due to the ergosphere. This spontaneous emission is closely related to the classical superradiance. It occurs only for modes $e^{-i \omega t} e^{i n \phi}$ satisfying $\omega / n<\Omega$ i.e., when the angular velocity of the mode is less than the angular velocity of the black hole. A rough estimate of the rate of mass loss due to radiation from the ergosphere is [16]

$$
\frac{d M}{d t} \sim \Omega^{2}
$$

Thus a rotating dilaton black hole will radiate away most of its angular momentum. The area of its event horizon will decrease and the potential barriers outside the horizon will grow.

Does a rotating dilaton black hole, at late times, resemble an elementary particle? If the black hole radiates away all its angular momentum before it approaches the extremal limit, then its evolution from that point will be the same as in the nonrotating case. If instead the black hole reaches the extremal limit before radiating away its angular momentum, then its thermal radiation (28) will vanish, but the radiation from the ergosphere (30) will become important. As more angular momentum is radiated away, the ergosphere radiation will increase, and so will the potential barriers outside the black hole. Thus the late stages of this evolution will resemble the situation described in [7]. Extremely high potential barriers surround a black hole which is radiating rapidly inside the barriers. In the rotating case the radiation is due to the ergosphere and in the nonrotating case the radiation is thermal. Thus an extremal black hole should behave in a similar fashion in particle scattering whether it has a small amount of angular momentum or not. However, one must keep in mind that in both cases the semi-classical description breaks down in the late stages of evaporation when the horizon becomes very small.

\section{Slowly Rotating Black Holes}

It is straightforward to solve the equations (3)-(5) to first order in the angular momentum parameter $a$. This is because most of the metric components depend only on $a^{2}$. Unfortunately, many of the interesting physical quantities also depend only on $a^{2}$, but we can still extract some useful information from the first order solution. 
Let us begin with the unrotating black hole for arbitrary $\alpha$, eq. (6), and consider the effect of adding a small amount of rotation $a$ to the black hole. We will discard any terms involving $a^{2}$ or higher powers in $a$. Inspection of the Kerr-Newman and Kaluza-Klein solutions shows that the only term in the metric that changes to $\mathcal{O}(a)$ is $g_{t \phi}$. Similarly, the dilaton does not change to $\mathcal{O}(a)$, and $A_{\phi}$ is the only component of the vector potential that changes to $\mathcal{O}(a)$. However, to this order, the change in $A_{\phi}$ is uniquely determined by the charge.

Thus, for arbitrary $\alpha$, we can assume the following form of the metric

$$
\begin{aligned}
d s^{2}= & -\left(1-\frac{r_{+}}{r}\right)\left(1-\frac{r_{-}}{r}\right)^{\frac{1-\alpha^{2}}{1+\alpha^{2}}} d t^{2}+\frac{d r^{2}}{\left(1-\frac{r_{+}}{r}\right)\left(1-\frac{r_{-}}{r}\right)^{\frac{1-\alpha^{2}}{1+\alpha^{2}}}}+r^{2}\left(1-\frac{r_{-}}{r}\right)^{\frac{2 \alpha^{2}}{1+\alpha^{2}}} d \Omega \\
& -2 a f(r) \sin ^{2} \theta d t d \phi,
\end{aligned}
$$

where $f(r)$ is a function to be determined. The dilaton and potential should be

$$
\Phi=\frac{\alpha}{1+\alpha^{2}} \log \left(1-\frac{r_{-}}{r}\right), \quad A_{t}=\frac{Q}{r}, \quad A_{\phi}=-a \sin ^{2} \theta \frac{Q}{r} .
$$

Using the linearized form of the equations of motion (3)-(5), we see that eqs. (31) and (32) are solutions as long as

$$
\begin{aligned}
f(r)= & \frac{r^{2}\left(1+\alpha^{2}\right)^{2}\left(1-\frac{r_{-}}{r}\right)^{\frac{2 \alpha^{2}}{1+\alpha^{2}}}}{\left(1-\alpha^{2}\right)\left(1-3 \alpha^{2}\right) r_{-}^{2}} \\
& -\left(1-\frac{r_{-}}{r}\right)^{\frac{1-\alpha^{2}}{1+\alpha^{2}}}\left(1+\frac{\left(1+\alpha^{2}\right)^{2} r^{2}}{\left(1-\alpha^{2}\right)\left(1-3 \alpha^{2}\right) r_{-}^{2}}+\frac{\left(1+\alpha^{2}\right) r}{\left(1-\alpha^{2}\right) r_{-}}-\frac{r_{+}}{r}\right) .
\end{aligned}
$$

In deriving $f(r)$, we have fixed an integration constant arising from the equations of motion so that $f(r) \rightarrow c / r$ as $r \rightarrow \infty$. This is the desired asymptotic behavior, and completely determines $f(r)$.

It is straightforward to verify that $f(r)$ agrees with the Kerr-Newman solution when $\alpha=0$, and with the Kaluza-Klein solution (19) when $\alpha=\sqrt{3}$ (after using (26), shifting $r \rightarrow r+r_{-}$and rescaling $a$ by a constant). At first glance, $f(r)$ appears to diverge in the string limit, $\alpha=1$. This is not actually the case; when $\alpha=1$,

$$
f(r)_{\alpha=1}=\frac{2 r^{2}}{r_{-}^{2}}\left(1-\frac{r_{-}}{r}\right) \log \left(1-\frac{r_{-}}{r}\right)-1+\frac{2 r}{r_{-}}+\frac{r_{+}}{r} .
$$

Similarly, $\alpha=1 / \sqrt{3}$ appears singular in the general solution (33), but is also well-behaved. Thus, $f(r)$ as given in eq. (33) is the correct solution to $\mathcal{O}(a)$ for all $\alpha$.

We argued earlier that for $\alpha>1$, a small amount of rotation should produce a large change in the geometry close to the horizon of a nearly extremal black hole. We can see 
this explicitly from (33). In the extremal limit, as $r$ approaches $r_{+}=r_{-}$, the second term in $f(r)$ diverges for $\alpha>1$. Thus adding even a little rotation drastically changes the unrotating solution near the horizon.

What else can we learn from the slowly rotating black hole? The surface gravity and area of the event horizon do not change to $\mathcal{O}(a)$. However, the angular momentum $J$ and the magnetic dipole moment $\mu$ first appear at this order. The angular momentum is related to the asymptotic form of the metric by

$$
g_{t \phi} \stackrel{r \rightarrow \infty}{\longrightarrow}-\frac{2 J}{r} \sin ^{2} \theta+\mathcal{O}\left(\frac{1}{r^{2}}\right)
$$

which gives

$$
J=\frac{a}{2}\left(r_{+}+\frac{3-\alpha^{2}}{3\left(1+\alpha^{2}\right)} r_{-}\right) .
$$

The magnetic moment is related to the asymptotic form of the vector potential by

$$
A_{\phi} \stackrel{r \rightarrow \infty}{\longrightarrow}-\frac{\mu \sin ^{2} \theta}{r}+\mathcal{O}\left(\frac{1}{r^{2}}\right)
$$

so that

$$
\mu=a Q
$$

If we define a parameter $g$ in the usual way by

$$
\mu=g \frac{Q J}{2 M}
$$

then we find

$$
g=2-\frac{4 \alpha^{2} r_{-}}{\left(3-\alpha^{2}\right) r_{-}+3\left(1+\alpha^{2}\right) r_{+}} .
$$

When $\alpha=0$, we recover the well known, but still remarkable fact that the Kerr-Newman black hole has $g=2$ just like the electron (up to quantum corrections). For $\alpha \neq 0, g$ still approaches 2 in the limit of small charge. However, as the charge increases, $g$ decreases to a minimum of $g=2-2 \alpha^{2} /\left(\alpha^{2}+3\right)$ for the extremal black hole. So for string theory with $\alpha^{2}=1, \frac{3}{2} \leq g \leq 2$ and for Kaluza-Klein theory with $\alpha^{2}=3,1 \leq g \leq 2$.

\section{Rotating Black Strings}

The Kaluza-Klein black hole was constructed by taking a four dimensional uncharged black hole, adding an extra dimension, Lorentz boosting, and then using a Kaluza-Klein reduction [8]. This procedure is very similar to the procedure recently used to generate axion charged black string solutions [11]. The only difference is that instead of using a Kaluza-Klein reduction in the last step, one uses a $\sigma$-model duality transformation [17]. 
This transformation relates solutions to the low energy action of string theory: eq. (1) with $\alpha=1$ plus a term involving the three form $H_{\mu \nu \rho}$ (which is the curl of a two form $B_{\mu \nu}$ ). Sigma model duality is most conveniently described in terms of the metric that the string directly couples to. This is equal to the metric appearing in (1) multiplied by a (dimension dependent) power of $e^{\Phi}$. In terms of the string metric, the (five dimensional) low energy string action is

$$
S=\int d^{5} x \sqrt{-g} e^{-2 \Phi}\left(-R-4(\nabla \Phi)^{2}+\frac{1}{12} H^{2}\right),
$$

where we have set the Maxwell field to zero.

To obtain rotating black string solutions to (41), begin with the Kerr black hole. Since $\Phi=0$, this is a solution to the string equations of motion. Add on an extra flat direction $x$, and Lorentz boost $t \rightarrow(t+v x) / \sqrt{1-v^{2}}, x \rightarrow(x+v t) / \sqrt{1-v^{2}}$. Now using the $\sigma$-model duality transformation, we obtain a five dimensional rotating, charged black string with metric

$$
\begin{aligned}
d s^{2}= & -\frac{1-Z}{B^{2}} d t^{2}-\frac{2 a Z \sin ^{2} \theta}{B^{2} \sqrt{1-v^{2}}} d t d \phi+\left[\left(r^{2}+a^{2}\right)+a^{2} \sin ^{2} \theta \frac{Z}{B^{2}}\right] \sin ^{2} \theta d \phi^{2} \\
& +\frac{\Sigma}{\Delta_{0}} d r^{2}+\Sigma d \theta^{2}+B^{-2} d x^{2}
\end{aligned}
$$

where $\Sigma, \Delta_{0}, Z$, and $B$ were defined previously, with a dilaton field

$$
\Phi=-\log B
$$

and antisymmetric tensor field

$$
B_{x t}=\frac{v}{\left(1-v^{2}\right)} \frac{Z}{B^{2}}, \quad B_{x \phi}=-a \sin ^{2} \theta \frac{v}{\sqrt{1-v^{2}}} \frac{Z}{B^{2}} .
$$

It is straightforward to use the higher dimensional generalizations of the Kerr solution [18] to derive rotating black strings in higher dimensions.

The properties of the rotating black string (42) are very similar to the Kaluza-Klein black hole. Indeed, if $d \widetilde{s}^{2}$ denotes the Kaluza-Klein metric (19), then the black string metric (42) is simply $d s^{2}=e^{\Phi} d \tilde{s}^{2}+e^{2 \Phi} d x^{2}$. The mass per unit length, axion charge per unit length and angular momentum per unit length are given by (23), (24) and (25). The rotating black string has an event horizon and nonsingular inner horizon at $r=$ $m \pm \sqrt{m^{2}-a^{2}}$, and a curvature singularity at $r=0, \theta=\pi / 2$. The surface gravity of the black string is equal to that of the Kaluza-Klein solution (28).

The extremal limit of the rotating black string is quite different from the extremal limit of the unrotating black string [11]. In the latter case, the extremal limit corresponds to the field outside a straight fundamental string [19], and is boost invariant in the $x$ - $t$ 
plane. The extremal limit of the rotating black string is not boost invariant, and it is not clear whether it can be interpreted as the field outside of an excited fundamental string.

Acknowledgements

We would like to thank D. Wiltshire for bringing [8], [13], and [14] to our attention. This work was supported in part by NSF Grant PHY-9008502.

\section{References}

1. P. Bizon, Phys. Rev. Lett. 64, 2844 (1990); M. Volkov and D. Gal'tsov, Sov. J. Nucl. Phys. 51, 1171 (1990); H. Kunzle and A. Masood-ul-Alam, J. Math. Phys. 31, 928 (1990).

2. P. Bizon and R. Wald, Phys. Lett. B267, 173 (1991).

3. Kimyeong Lee, V. Nair and E. Weinberg, "Black Holes in Magnetic Monopoles," Columbia preprint CU-TP-539, December 1991.

4. G. Gibbons and K. Maeda, Nucl. Phys. B298, 741 (1988).

5. D. Garfinkle, G. Horowitz and A. Strominger, Phys. Rev. D43, 3140 (1991).

6. J. Preskill, P. Schwarz, A. Shapere, S. Trivedi, and F. Wilczek, Mod. Phys. Lett. A6, 2353 (1991).

7. C. Holzhey and F. Wilczek, "Black Holes as Elementary Particles," IAS preprint IASSNS-HEP-91/71, December 1991.

8. V. Frolov, A. Zelnikov, and U. Bleyer, Ann. Phys. (Leipzig) 44, 371 (1987).

9. G. Horowitz and A. Strominger, Nucl. Phys. B360, 197 (1991).

10. J. Horne and G. Horowitz, Nucl. Phys. B368, 444 (1992).

11. J. Horne, G. Horowitz, and A. Steif, Phys. Rev. Lett. 68, 568 (1992).

12. R. Wald, Gereral Relavitity (U. of Chicago Press, Chicago), 1984.

13. H. Leutwyler, Arch. Sci. 13, 549 (1960); P. Dobiasch and D. Maison, Gen. Rel. Grav. 14, 231 (1982); A. Chodos and S. Detweiler, Gen. Rel. Grav. 14, 879 (1982); G. Gibbons and D. Wiltshire, Ann. Phys. 167, 201 (1986), erratum, ibid. 176 (1987) 393.

14. G. Clément, Gen. Rel. Grav. 18, 137 (1986). 
15. D. Page, Phys. Rev. D14, 3260 (1976).

16. W. Unruh, Phys. Rev. D10, 3194 (1974).

17. T. Buscher, Phys. Lett. B201, 466 (1988); Phys. Lett. B194, 59 (1987).

18. R. Myers and M. Perry, Ann. Phys. 172, 304 (1986).

19. A. Dabholkar, G. Gibbons, J. Harvey and F. Ruiz, Nucl. Phys. B340, 33 (1990). 